Junior MARSIPAN Guidelines, is proposed to improve initial assessment, inpatient management of refeeding risks and to plan discharge. It is hoped this will lead to safer, more supportive and seamless care for young people with eating disorders when they become medically unstable.

\section{GP294 JUNIOR CLINICIANS' EXPERIENCES IN LEADING IMPLEMENTATION OF NEW MODELS OF CARE: DESIGNING AND DEVELOPING A PAEDIATRIC HOSPITAL AT HOME SERVICE}

Shruti Patel ${ }^{*}$, Alice Stebbings. Chelsea and Westminster NHS Trust, London, UK

\subsection{6/archdischild-2019-epa.353}

Promoting care closer to home for children has been a policy objective for some time and has been reiterated in NHS England's 10 year plan. Models delivering acute care outside the hospital across Europe have shown to deliver equivalent clinical outcomes whilst reducing ED re-attendance and length of stay. Junior clinical staff often rotate through multiple hospitals and as such can share best practice across organisations. However, too often leadership structures are not set-up to nurture, support or empower junior clinicians to enact change. In the context of worsening morale of the junior medical workforce as well as inner city difficulties in retention of junior nurses, providing opportunities to lead and participate in service development can increase engagement.

We report experiences of designing and developing a paediatric hospital at home service with a focus on recruitment and retention. The service was pitched to executive level by a junior doctor, with planning and design co-led with a junior charge nurse, supported by a team of consultants, senior matrons and operational leads. The junior clinicians led design workshops, wrote a funding bid and drafted the service specification.

Design workshops engaged doctors, nurses from both ED and ward backgrounds as well as parents and a wide range of professionals. Each was asked to bring a junior colleague and subsequently interviewed on their experiences of engaging in service design. For the majority of participants this was their first experience of service design, all felt more likely to engage in future discussions about the service and more invested in working in the service. Senior colleagues reported contributions of junior colleagues as valuable by providing a different perspective, including adding a health promotion aspect.

Junior clinicians can be well placed to lead new service development, particularly in a multi-professional partnership. As well as delivering improvement in quality of care, there are retention benefits to the workforce in enabling leadership roles and opportunities to engage in service development. Adequate support from senior leaders and engagement of wider team are critical enablers.

\section{GP295 HAEMOPHILUS INFLUENZA (HI)EARLY ONSET SEPSIS CASE CLUSTER: A CASE REPORT}

${ }^{1}$ Mugahid Ibrahim, ${ }^{2}$ Qasim Mahmood, ${ }^{1}$ Muhammad Zia*, 'Ilatadar Hussein, 'Khorshed Khalifa, 'Naula O'Connell. 'University Maternity Hospital Limerick, Limerick, Ireland; ${ }^{2}$ Kerry General Hospital, Tralee, Ireland

10.1136/archdischild-2019-epa.354
Background To describe two cases of neonatal (HI) early onset septicaemia.

Case 1 Female infant born at term to a 25 years old primigravida. Mother had pyrexia one hour post-delivery. Septic screen performed and IV antibiotics started. Afterwards, mother remained clinically well and no organisms were isolated in her blood culture. At $12 \mathrm{hrs}$ of age the newborn developed pyrexia, poor feeding and hypertonia. Full septic screen was obtained and antibiotics were started. Bloods showed leucopenia, neutropenia and high CRP, and her blood culture grew $H$. influenzae $(\mathrm{HI})$ at 48 hours of age for which antibiotics were adjustment according to sensitivity. PCR test confirmed non-type able $H$. influenzae (NTHi). Antibiotic course completed and follow up at 2 months showed normal growth development.

Case 226 years old presented with reduced fetal movement and light vaginal bleeding. Bloods showed leucocytosis \& neutrophilia. Chorioamnionitis suspected and antibiotics commenced. Her blood culture grew (HI) at 31 hours post incubation with no growth reported on her HVS or MSU. Six hours later, a baby boy was delivered at term. He was admitted to NICU for intermittent grunting. Septic screen performed and antibiotics started and further adjusted according to sensitivity. Only blood PCR test detected (HI) type B (Hib) with no growth on blood culture. He was discharged home in a good condition after antibiotics course completed. Placental swabs showed $(H I)$ on the placental fetal surface.

Discussion (HI) should be considered as a potential maternal, fetal and neonatal pathogen. However, HI infection became much less since the introduction of Hib vaccine. (HI) growth in maternal vaginal swabs should always be reported by the laboratory to the requesting clinician. Specimens collected from the placenta or vagina of pregnant mothers showing signs of premature rupture of the membranes, chorioamnionitis and antepartum or post-partum sepsis should be inoculated onto agar selective for $(\mathrm{HI})$, in order to ensure recovery of this pathogen. (NTHi) have been recognized as obstetrics and gynecology pathogens. Since its significant morbidity and mortality, incorporating a screening protocol to detect colonization may have beneficial effects.

\section{GP296 EPILEPSY \&US - GIVING A REAL VOICE TO CHILDREN, YOUNG PEOPLE AND FAMILIES AS PART OF A NATIONAL AUDIT PROGRAMME}

Calvin Down*, Emma Sparrow, Colin Dunkley. Royal College of Paediatrics and Child Health, London, UK

\subsection{6/archdischild-2019-epa.355}

Epilepsy12 was established in 2009 with the aim of helping epilepsy services to measure and improve care for children and young people with seizures and epilepsies across England and Wales. Epilepsy12 is delivered by the Royal College of Paediatrics and Child Health.

For Round 2 of the audit, children and young people with epilepsy, and their parents/carers, were invited to complete a Patient Reported Experience Measure (PREM) questionnaire on their experiences of care from their epilepsy service over the previous year.

2,335 questionnaires were returned. Some findings were:

- $88 \%$ were satisfied with overall care from their epilepsy service 